\title{
Gene ontology enrichment and network analysis for differently expressed genes related to aggressive behavior
}

\author{
Tiys E. ${ }^{1}$, Chadaeva I. ${ }^{1,2 *}$, Oshchepkov D. ${ }^{1}$, Shichevich S. ${ }^{1}$, Kozhemyakina R. ${ }^{1}$ \\ ${ }^{1}$ Institute of Cytology and Genetics, SB RAS, Novosibirsk, Russia \\ ${ }^{2}$ Novosibirsk State University, Novosibirsk, Russia \\ *e-mail: ichadaeva@bionet.nsc.ru
}

Key words: differently expressed genes, behaviour, tame rats, aggressive rats, ANDVisio, FunGeneNet

Motivation and Aim: The purpose of this work was to analyze the co-expression, possible physical interactions, metabolic pathway, and co-localization of genes significantly different in expression levels between animals with contrasting behavioral phenotypes, gray rats, selected for tame and aggressive behavior for over 40 years (since 1972, more than 90 generations) [1].

Methods and Algorithms: Using bioinformatic analysis of RNA sequencing data, lists of differentially expressed genes for several brain regions of aggressive and tame rats (hippocampus, hypothalamus, mesencephalic tegmentum and periaqueductal gray) were compiled. Using the gene network research programs ANDSystem [2] and FunGeneNet [3], we analyzed 78 genes, the expression level of which was statistically different.

Results: The search for overrepresented processes using PANTHER and the analysis of networks for groups of these genes using FunGeneNet did not reveal a significant excess of the number of intergenic links in comparison with random networks.

Conclusion: The results of our analysis can be interpreted as an evidence of a known phenomenon, the polygenic determination of behavioral phenotype. In our case, these phenotypes are "aggressiveness towards a human" and "non-aggressiveness towards a human" in the rats of the studied lines. The resulting absence of direct connections between genes with the differential expression in the tame and aggressive rats confirms our assumption about a nonlinear interaction of these genes and their complex coordination in the manifestation of hereditarily fixed tame or aggressive behavior. Further work is planned to identify links of the differentially expressed genes.

Acknowledgements: The research is supported by RFBR (grant 18-34-00496).

\section{References}

1. Belyaev D.K., Borodin P.M. The influence of stress on variation and its role in evolution. Biol. Zent. 1982;100:705-714.

2. Ivanisenko V.A. et al. ANDSystem: an associative network discovery system for automated literature mining in the field of biology. BMC Syst Biol. 2015;9(Suppl. 2):S2.

3. Tiys E.S. et al. FunGeneNet: a web tool to estimate enrichment of functional interactions in experimental gene sets. BMC Genomics. 2018;19:76. 